\title{
In situ measurements of density fluctuations and compressibility in silica glasses as a function of temperature and thermal history
}

\author{
C. Levelutt and A Faivre, and R. Le Parc \\ Laboratoire des Colloides, Verres et Nanomatériaux, \\ CNRS/UMR558\%, Université Montpellier II, \\ cc 69, 34095 Montpellier cedex, France \\ B. Champagnon \\ Laboratoire de Physicochimie des Matériaux Luminescents, \\ Université Lyon 1, CNRS/UMR 5620, 69622 Villeurbanne Cedex \\ J.-L. Hazemann \\ Laboratoire de Cristallographie, UPR 5031, 26 avenue des Martyrs, \\ BP 166, 38042 Grenoble CEDEX 9, France and LGIT, \\ CNRS/UMR 5559, Université Joseph Fourier, \\ 38402 Saint Martin d'Hères, France \\ J.-P. Simon \\ Laboratoire de Thermodynamique et Physicochimie Métallurgiques, \\ UMR 5614, INP et UJF Grenoble, BP 75, \\ 38402 Saint Martin d'Hères, France
}

(Dated: November 7, 2018) 


\begin{abstract}
In this paper, small-angle X-ray scattering measurements are used to determine the different compressibility contributions, as well as the isothermal compressibility, $\chi_{T}^{0}$, in thermal equilibrium in silica glasses having different thermal histories. Using two different methods of analysis, in the supercooled liquid and in the glassy state, we obtain respectively the temperature and fictive temperature dependences of $\chi_{T}^{0}$. The values obtained in the glass and supercooled liquid states are very close to each other. They agree with previous determinations of the literature. The compressibility in the glass state slightly decreases with increasing fictive temperature. The relaxational part of the compressibility is also calculated and compared to previous determinations. We discussed the small differences between the different determinations.

PACS numbers: 61.43.Fs, 61.10.Eq, 51.35.+a, 64.70.Pf
\end{abstract}




\section{INTRODUCTION}

The nanometer range in glasses is expected to correspond to fluctuations of density and composition, whereas at shorter scale the structure is very close to that of the crystalline counterpart and at larger scale the medium can be considered as homogeneous. Those fluctuations, or part of them, are frozen-in during the cooling process and thus they are determined by the cooling rate and the thermal history of the glass sample, which can be characterized by a "fictive temperature" for a certain property (see Ref. 1 for more details). As a consequence, the fluctuations are expected to depend on the fictive temperature which will be used in this paper to characterize the thermal history of each glass sample.

One possible experimental method to study electronic density fluctuations at the nanometer scale is Small-Angle X-ray Scattering (SAXS), which is sensitive to both composition and density fluctuations. Silica glass is a good candidate to study density fluctuations as composition fluctuations do not occur in this "single component" glass (made of $\mathrm{SiO}_{2}$ "molecules"). It represents a material of significant commercial as well as fundamental interest. The characterization of density fluctuations in silica is of great importance for the fundamental understanding of the glass transition but also for commercial applications, such as for silica-based optical fibers.

Density fluctuations are related to thermodynamics parameters such as compressibility of the sample. Small angle x-ray scattering intensity measurements in silica glass across the glass transition temperature give access to the isothermal compressibility. It can be calculated in the melt, as well as in the glassy state where it depends on the thermal history of the sample. In the glassy state, the isothermal compressibility can be decomposed into different terms contributing to frozen-in or still propagating density fluctuations.

In this paper we will use SAXS intensity measured both as a function of temperature

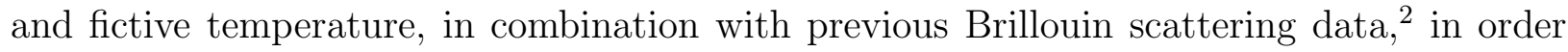
to determine the different compressibility contributions to the density fluctuations, both above and below the glass transition temperature. By a full point by point analysis, we

obtain the temperature and fictive temperature variations of theses different compressibility contributions. The results are discussed in comparison with previous measurements. 


\section{THEORETICAL BACKGROUND}

The theoretical description of the SAXS intensity for one composition unit or molecule, $I(q)$, due to density fluctuations is rather well-known in liquids. Thermodynamic fluctuations theory predicts that:

$$
I(q=0)=\frac{N_{a} \times\left(\sum Z\right)^{2} \times \rho_{0}}{m} \times k_{b} T \chi_{T}^{0}(T),
$$

where $N_{a}$ is Avogadro's number, $\sum Z$ the total number of electrons per molecule, $m$ the

molar mass of one molecule, $\chi_{T}^{0}(T)$ the isothermal compressibility of the liquid, $\rho_{0}$ the density and $k_{b}$ the Boltzman's constant. A similar equation can be found for the low $q$ limit of the static structure factor:

$$
S(q=0)=n \times k_{b} T \chi_{T}^{0}(T)=\frac{N_{a} \times N \times \rho_{0}}{m} k_{b} T \chi_{T}^{0}(T)
$$

where $S(q)$ is the static structure factor, $n$ is the number density of atoms and $N$ the number of atoms in the molecule. ${ }^{3}$ Then $S(q=0)$ is related to the intensity $I(q=0)$ per molecule by $S(q=0)=N I(q=0) /\left(\sum Z\right)^{2}$.

In the glassy state, equation (11) is no more valid because the system is out of thermodynamic equilibrium. Historically, several different descriptions have been proposed to relate the low $q$ SAXS or light scattering intensity to compressibilities in the glassy state.

Weinberg first proposed that all density fluctuations are frozen-in at $T_{g}$, and thus, equation (11) becomes independent of the temperature:

$$
I(q=0)=\frac{N_{a} \times\left(\sum Z\right)^{2} \times \rho_{0}}{m} \times k_{b} T_{g} \chi_{T}^{0}\left(\mathbf{T}_{\mathbf{g}}\right) .
$$

Wendorff rather said that below the glass transition, $\frac{5}{,}$

$$
I(q=0)=\frac{N_{a} \times\left(\sum Z\right)^{2} \times \rho_{0}}{m} \times k_{b} \mathbf{T} \chi_{T}^{0}\left(T_{g}\right)
$$

which yields to density fluctuations which are still decreasing with decreasing temperature, but should go to zero at absolute zero temperature.

Roe and Curro mentioned that below the glass transition temperature, the density fluctuations amplitude is much higher than that expected from (44). They assumed that the density fluctuations can be decomposed into two components: one dynamic contribution, related to the isothermal compressibility in the glass, which is determined by equation (4) ${ }^{6.7}$ 
and one static or quasi-static term, $I(q=0, T=0)$, which represents the density fluctuations that are frozen-in. This static contribution can be attributed to structural entities whose relaxation times are longer than experimental time scale.

$$
\begin{gathered}
I(q=0)=I(q=0, T=0)+\frac{N_{a} \times\left(\sum Z\right)^{2} \times \rho_{0}}{m} k_{b} \mathbf{T} \chi_{T}^{0}\left(T_{g}\right) \\
\text { static } \\
\text { dynamic }
\end{gathered}
$$

On another hand, Ruland and coworkers decomposed the density fluctuation below $T_{g}$ in one component due to frozen-in disorder, independent of temperature, $I(q=0, T=0)$, and one temperature dependent component due to pressure fluctuations (longitudinal elastic waves),

$$
I(q=0)=I(q=0, T=0)+\frac{N_{a} \times\left(\sum Z\right)^{2} \times \rho_{0}}{m} k_{b} T\left\langle\frac{1}{\rho V_{l, \infty}^{2}}\right\rangle
$$

where $V_{l, \infty}$ is the longitudinal sound wave velocity and \langle\rangle indicates a spatial average.. .9

The same kind of decomposition has been introduced by Laberge using in equation (1) the adiabatic compressibility, $\chi_{S}^{0}: \chi_{T}^{0}=\left(\chi_{T}^{0}-\chi_{S}^{0}\right)+\chi_{S}^{0}$. The fluctuations can be separated in isobaric fluctuations, related to $\left(\chi_{T}^{0}-\chi_{S}^{0}\right)$ and adiabatic fluctuations, related to $\chi_{S}^{0}{ }^{10}$

The adiabatic fluctuations consist of sound waves and variations in the local structure. Laberge ${ }^{10}$ introduced a term of relaxational origin in the density fluctuations by pointing out that if the material is viscous enough so that the structural relaxation is slow compared to the oscillations of infinite frequency acoustic waves, then, the phonons contribution is defined by the infinite frequency compressibility $\chi_{S}^{\infty}$ and the residual part by the relaxational compressibility $\chi_{S}^{r}=\chi_{S}^{0}-\chi_{S}^{\infty}$. These assumptions lead to the following equation:

$$
I(q=0)=\frac{N_{a} \times\left(\sum Z\right)^{2} \times \rho_{0}}{m} k_{b} T\left[\left(\chi_{T}^{0}-\chi_{S}^{0}\right)+\chi_{S}^{r}+\chi_{S}^{\infty}\right]
$$

with:

$$
\chi_{S}^{\infty}=1 /\left(\rho\left(V_{l, \infty}\right)^{2}-4 \rho\left(V_{t, \infty}\right)^{2} / 3\right)=1 /\left(M_{\infty}-4 G_{\infty} / 3\right)=1 / K_{\infty},
$$

where $V_{t, \infty}$ is the high frequency transverse sound velocity, $G_{\infty}=\rho\left(V_{t, \infty}\right)^{2}$ is the high frequency shear modulus, $M_{\infty}=\rho\left(V_{l, \infty}\right)^{2}$, the high frequency longitudinal modulus and $K_{\infty}$ is the infinite frequency compression modulus.

He, moreover, replaced $\chi_{S}^{\infty}$ by $M_{\infty}^{-1}$, and $\chi_{S}^{r}$ by $\chi_{S}^{0}-M_{\infty}^{-1}$, considering that this is more appropriate in a viscoelastic material capable of supporting high-frequency shear stress. The scattered amplitude due to density fluctuations then becomes: 


$$
\frac{I(q=0)}{N_{v} \times\left(\sum Z\right)^{2}}=k_{b} T\left[\left(\chi_{T}^{0}(T)-\chi_{S}^{0}(T)\right)+\left(\chi_{S}^{0}(T)-M_{\infty}^{-1}(T)\right)+M_{\infty}^{-1}(T)\right],
$$

with $N_{v}=N_{a} \times \rho_{0} / m$. This equation is valid for a viscoelastic medium in equilibrium at temperature $T$.

In the glassy state, Laberge considered that the two first terms, describing nonpropagating fluctuations, are kinetically arrested at a temperature in the annealing range because their relaxation time becomes considerably longer than the experimental time, as temperature is lowered. He assumed that these frozen-in fluctuations are the equilibrium fluctuations present in the glass at the fictive temperature $T_{f}$, thus giving:

$$
\frac{I(q=0)}{N_{v}\left(\sum Z\right)^{2}}=k_{b} T_{f}\left[\chi_{T}^{0}\left(T_{f}\right)-\chi_{S}^{0}\left(T_{f}\right)\right]+k_{b} T_{f}\left[\chi_{S}^{0}\left(T_{f}\right)-M_{\infty}^{-1}\left(T_{f}\right)\right]+k_{b} T M_{\infty}^{-1}(T) .
$$

term 1

term2

term 3

The third term is of vibrational origin and is identical to the phonon term in equation (6). The first two terms are frozen-in. It can be noted that $T_{g}$ or $T_{f}$ are used indifferently to characterize the temperature at which a glass is frozen-in. In the following, we use $T_{f}$ for samples with a known thermal history (annealed and equilibrated at $T_{f}$ ) and $T_{g}$ by reference to a given composition when the thermal history is not known. The temperature dependence below the glass transition is related to term 3, proportional to temperature.

The fluctuations at the nanometer scale can also be probed by light scattering. Using Brillouin light scattering, the first two terms occurs in the elastic part $i$. e. the Rayleigh part, whereas the third one, related to the longitudinal velocity of sound arises from propagating fluctuations and then contributes to the inelastic (Brillouin) intensity. Thus, the ratio of the static to propagating intensities, also called the Landau Placzek ratio, $R_{L P}$, gives access to the ratio of the first two terms over the third. In a viscoelastic liquid, $\frac{11}{1}$ using equation (11):

$$
R_{L P}(T)=\frac{\chi_{T}^{0}(T)-M_{\infty}^{-1}(T)}{M_{\infty}^{-1}(T)}=\chi_{T}^{0}(T) M_{\infty}(T)-1
$$

In a glass, ${ }^{12}$, equation (10) yield:

$$
R_{L P}(T)=M_{\infty}(T)\left[\chi_{T}^{0}\left(T_{f}\right)-M_{\infty}^{-1}\left(T_{f}\right)\right] \frac{T_{f}}{T}
$$




\begin{tabular}{|c|c|c|c|c|}
\hline authors & $T$ or $T_{f}$ & $\chi_{T}^{0}(T$ or $)\left(\mathrm{m} \mathrm{s}^{2} \mathrm{~kg}^{-1}\right)$ & $\chi_{S}^{r}\left(\mathrm{~m} \mathrm{~s}^{2} \mathrm{~kg}^{-1}\right)$ & $\chi_{S}^{\infty}\left(\mathrm{m} \mathrm{s}^{2} \mathrm{~kg}^{-1}\right)$ \\
\hline Laberge et $a^{10}$ & $T_{f}=1600 \mathrm{~K}$ & $7.3 \pm 0.6 \times 10^{-11}$ & & \\
\hline Bucaro et a ${ }^{11}$ & $T=1400-1700 \mathrm{~K}$ & $8.5 \pm 0.3 \times 10^{-11}$ & $6.3 \times 10^{-11}$ & $2.2 \times 10^{-11}$ \\
\hline Krol et al12 & $T_{f}=1273 \mathrm{~K}$ & $7.72 \times 10^{-11}$ & $5.68 \times 10^{-11}$ & $2.04 \times 10^{-11}$ \\
\hline Watanabe et $a^{13}$ & $T_{f}=1230-1670 \mathrm{~K}$ & $10.5 \times 10^{-11}$ & $6.4 \times 10^{-11}$ & $4.1 \times 10^{-11}$ \\
\hline Polian et al 14 & $T=1370 \mathrm{~K}$ & & & $2.23 \times 10^{-11}$ \\
\hline this work and ${ }^{2}$ & $T_{f}=1373 \mathrm{~K}$ & $6.16 \pm 0.24 \times 10^{-11}$ & $3.88 \pm 0.25 \times 10^{-11}$ & $2.28 \pm 0.07 \times 10^{-11}$ \\
\hline this work and ${ }^{2}$ & $T_{f}=1773 \mathrm{~K}$ & $5.69 \pm 0.23 \times 10^{-11}$ & $3.46 \pm 0.22 \times 10^{-11}$ & $2.22 \pm 0.07 \times 10^{-11}$ \\
\hline
\end{tabular}

TABLE I: Comparison of isothermal compressibility, adiabatic relaxational compressibility and adiabatic infinite frequency compressibility taken from the literature.

\section{PREVIOUS MEASUREMENTS}

There are relatively few results about the compressibility in silica and especially few results concerning its temperature and fictive temperature dependence. Most of them use light scattering. The different compressibility values quoted in the literature are reported in table

Bucaro et Dardy used Brillouin light scattering and measurements of the Landau Placzek ratio to determine the isothermal compressibility $\chi_{T}^{0}$ at high temperature, between 1400 and $1700 \mathrm{~K}$, using equation (11) ${ }^{11}$ This equation is only valid in the (viscous) liquid. They found $\chi_{T}^{0}=8.5 \pm 0.3 \times 10^{-11} \mathrm{~m} \mathrm{~s}^{2} \mathrm{~kg}^{-1}$ with no temperature dependence in the range where $\chi_{T}^{0}$ was determined. They also deduced the compressibility at infinite frequency $\chi_{S}^{\infty}$ directly from the longitudinal and transverse Brillouin shifts; they obtained about $2.2 \times 10^{-11} \mathrm{~m} \mathrm{~s}^{2} \mathrm{~kg}^{-1}$ in the same temperature range.

Krol et al12 also measured the Landau Placzek ratio as well as Brillouin shifts (shear and longitudinal modulus) as a function of temperature in a silica glass with a fictive temperature equal to $1273 \mathrm{~K}$. They used equation (12), taking into account the variation of the high frequency modulus with temperature. From the slope of $R_{L P}(T) / M_{\infty}(T)$, plotted below the glass transition as a function of $1 / T$, they deduced $\chi_{T}^{0}\left(T_{f}\right)$. Calculating the infinite frequency adiabatic compressibility from both longitudinal and transverse Brillouin shifts, $\chi_{S}^{\infty}=\left(M_{\infty}-\frac{4}{3} G_{\infty}\right)^{-1}$, they deduced the relaxational contribution at $T_{f}, \chi_{S}^{r}\left(T_{f}\right)=\chi_{S}^{0}-$ 
$\chi_{S}^{\infty} \simeq \chi_{T}^{0}-\chi_{S}^{\infty}$ because in silica $\chi_{S}^{0}-\chi_{T}^{0}$ is negligible. Indeed, it is related to thermal expansion, $\underline{\underline{10}}$ which is very low in silica glass: $\chi_{T}^{0}\left(T_{f}\right)-\chi_{S}^{0}\left(T_{f}\right)=\frac{\alpha\left(T_{f}\right)^{2} \times T_{f}}{\rho_{0} \times C_{p}\left(T_{f}\right)}, T_{f}=1273 \mathrm{~K}$, $\alpha\left(T_{f}\right)=0.5 \times 10^{-6} \mathrm{~K}^{-1}$ (Ref. 15), $\rho_{0}=2202 \mathrm{~kg} \mathrm{~m}^{-3}$, and $C_{p}\left(T_{f}\right)=1231 \mathrm{~m}^{2} \mathrm{~s}^{-2} \mathrm{~K}^{-1}$. These values yield to $\chi_{T}^{0}\left(T_{f}\right)-\chi_{S}^{0}\left(T_{f}\right)=1.08 \times 10^{-16} \mathrm{~m} \mathrm{~s}^{2} \mathrm{~kg}^{-1}$ Then, Krol et al obtained $\chi_{T}^{0}\left(T_{f}\right)=7.72 \times 10^{-11} \mathrm{~m} \mathrm{~s}^{2} \mathrm{~kg}^{-1}$, and $\chi_{S}^{\infty}=2.04 \times 10^{-11} \mathrm{~m} \mathrm{~s}^{2} \mathrm{~kg}^{-1}$, from which they deduce $\chi_{S}^{r} \simeq 5.68 \times 10^{-11} \mathrm{~m} \mathrm{~s}^{2} \mathrm{~kg}^{-1}$.

Saito et al $\frac{16.17}{}$ deduced the isothermal compressibility from the light scattering intensity, $I_{\text {light }}$, as a function of temperature. They used equation (10) in the liquid and the following expression in the glass:

$$
I_{\text {light }} \simeq I(q=0) \propto \chi_{T}^{r}\left(T_{f}\right) k T_{f}+\chi_{S}^{\infty}(T) k T,
$$

where $\chi_{T}^{r}=\chi_{T}^{0}-\chi_{T}^{\infty}$. This equation differs from equation (10) by the use of $\chi_{S}^{\infty}$ instead of $M_{\infty}^{-1}$ as the temperature-dependent part, the shear term being small enough to be neglected according to the authors. Another difference with equation (101) is the use of $\chi_{T}^{0}-\chi_{T}^{\infty}$ instead of $\chi_{T}^{0}-\chi_{S}^{\infty}$. They found a sudden increase of the isothermal compressibility at the glass transition and at the melting temperature, with no measurable temperature dependence within the glassy and supercooled liquid state $\frac{16}{\underline{16}}$ They found $\chi_{T}^{0}$ around $1.8 \times 10^{-11} \mathrm{~m} \mathrm{~s}^{2} \mathrm{~kg}^{-1}$ in the glass, and around $8 \times 10^{-11} \mathrm{~m} \mathrm{~s}^{2} \mathrm{~kg}^{-1}$ in the supercooled liquid, which yields to $\chi_{T}^{r}$ around $6 \times 10^{-11} \mathrm{~m} \mathrm{~s}^{2} \mathrm{~kg}^{-1} \cdot \underline{17}$

Apart from Saito's measurements, most compressibility measurements as a function of temperature across the glass transition concern the infinite frequency compressibility. $\chi_{S}^{\infty}$ has been measured using Brillouin light scattering by Polian et a $\mathrm{l}^{14}$ as a function of temperature using Brillouin scattering measurements of the longitudinal and transverse modes. They observed that $\chi_{S}^{\infty}$ decreases continuously as a function of temperature from about $2.7 \times$ $10^{-11} \mathrm{~m} \mathrm{~s}^{2} \mathrm{~kg}^{-1}$ at $400 \mathrm{~K}$ to $2.05 \times 10^{-11} \mathrm{~m} \mathrm{~s}^{2} \mathrm{~kg}^{-1}$ at $2300 \mathrm{~K}$. Those values are in agreement with high frequency previous data from several sources $\frac{18.19 .20}{2}$ compiled by Vukcevitch. $\frac{21}{\underline{T}}$ The data of Polian are also consistent with our determination of $\chi_{S}^{\infty}$ as a function of temperature. $\stackrel{2}{=}$

Concerning the effect of fictive temperature, Champagnon et al22 measured the Landau Placzek ratio at room temperature in several silica samples of same origin but prepared with different fictive temperatures from $T_{f}=1373 \mathrm{~K}$ to $T_{f}=1773 \mathrm{~K}$, using heat-treatment in the glass transition region (transformation range). They observed an increase of the $R_{L P}$ at room temperature as a function of fictive temperature. The increase is approximately 
linear above $T_{f}=1473 \mathrm{~K}$. From relation (12), one can deduce that $\chi_{T}^{0}\left(T_{f}\right)$ and $M_{\infty}^{-1}\left(T_{f}\right)$ vary slowly with $T_{f}$ between 1473 and $1773 \mathrm{~K}$.

There is one paper where compressibility is determined using SAXS intensity measurements at room temperature in four silica samples with different fictive temperatures. 13 The authors obtained a linear variation of the SAXS intensity as a function of fictive temperature. Following equation (13), they use a linear regression and obtained $\chi_{S}^{\infty}$ assumed to be independent of temperature, from the slope, and $\chi_{T}^{r}$ at the fictive temperature, from the intercept. They obtained $\chi_{S}^{\infty}=4.1 \times 10^{-11} \mathrm{~m} \mathrm{~s}^{2} \mathrm{~kg}^{-1}$ and $\chi_{T}^{r}\left(T_{f}\right)=6.4 \times 10^{-11} \mathrm{~m} \mathrm{~s}^{2} \mathrm{~kg}^{-1}$ for $\chi_{T}^{r}$. They add both terms to determine the adiabatic compressibility $\chi_{T}^{0} \simeq \chi_{T}^{r}+\chi_{S}^{\infty}=10.5 \times 10^{-11} \mathrm{~m}$ $\mathrm{kg}^{-1} \mathrm{~s}^{2}$.

\section{MEASUREMENTS}

We analyzed high purity silica samples (with very low $\mathrm{OH}$ content), all from the same batch. They are fusion glasses of type I 15 The different samples have different well-defined thermal histories. The temperature, time and conditions of annealing are reported on table III along with the quenching conditions. Annealing times are estimated from the expected relaxation times at the temperature of the heat-treatment, and the quenching rate (specially for the high temperature treatments) are assumed to be fast enough to preserve the high temperature structure. We plotted several spectroscopic features based on infrared or Raman measurements (position or intensities of peaks) as a function of annealing temperature. ${ }^{23.24}$ Those spectroscopic features have been previously reported to be attributed to structural characteristics sensitive to the fictive temperature, and to vary linearly with the inverse fictive temperature ${ }^{25,26}$ A linear relation was found for the six annealed samples, using the temperature of treatment as fictive temperature. Thus, we assumed that all the samples are treated long enough and quenched fast enough to be stabilized at their annealing temperature. In other words, the fictive temperature is assumed to be equal to the annealing temperature. Moreover, the sound velocity, measured using Brillouin scattering vary also linearly, as plotted versus the fictive temperature, in agreement with the hypothesis that samples are stabilized.

The small-angle x-ray measurements were performed on the D2AM experimental set-up of the European Synchrotron Radiation Facility (ESRF) at Grenoble, France. The mea- 


\begin{tabular}{cccc}
\hline \hline & & & Quench \\
temperature & time & conditions & conditions \\
\hline $1373 \mathrm{~K}$ & $60 \mathrm{~h}$ & in air & in air on a metallic plate \\
$1473 \mathrm{~K}$ & $1 \mathrm{~h}$ & in air & in air on a metallic plate \\
$1573 \mathrm{~K}$ & $1 \mathrm{~h} 45$ & in dry air & quenched in water \\
$1773 \mathrm{~K}$ & $1 \mathrm{~h} 45$ & in dry air & quenched in water \\
\hline \hline
\end{tabular}

TABLE II: Experimental conditions of annealing and quenching of the silica samples.

surements were performed on small plates of about $1 \mathrm{~mm}$ thickness. The data on silica samples were measured with an incident energy of $18 \mathrm{keV}$, yielding to an available $q$-range of $0.02-1 \AA^{-1}$, where $q=4 \pi \lambda^{-1} \sin \theta$ is the scattering vector, and $\theta$ is half of the scattering angle. The accumulation times were of $200 \mathrm{~s}$, and the data were collected using a CCD camera. In situ temperature measurements were performed using a high temperature molybdenum furnace already referred to in Ref. 27. The use of this furnace produces a reduction of the available $q$-range, which is about $0.02-0.7 \AA^{-1}$. The temperatures in the furnace were calibrated by observing the melting of a gold foil, which deviates from less than $2 \mathrm{~K}$ from the standard value. Radial integration and corrections from cosmic rays have been performed in the usual way. The x-ray was monitored before the furnace, $m_{0}$, and after the furnace, $m_{1}$, by scattering part of the beam with Kapton foils onto scintillation counters. Before measuring a new sample, the SAXS signal of the empty furnace, $I^{B}(q)$, was systematically measured. This background originated mainly from the Kapton windows and air scattering.

Based on the measured signal with the sample in place, $I^{S+B}(q)$, the signal originating from the sample was calculated according to

$$
I(q) \propto(t \ln 1 / t)^{-1}\left[I^{S+B}(q) / m_{1}^{S+B}-I^{B}(q) / m_{1}^{B}\right] m_{1}^{S+B} / m_{0}^{S+B},
$$

where the transmission factor $t=m_{1}^{S+B} m_{0}^{B} /\left(m_{0}^{S+B} m_{1}^{B}\right)$ allows for changes in the thickness and orientation of the sample. Doing this, the signal is corrected from the variations of the incident flux, as well as normalized to the absorption of the sample. The absolute intensity of the samples is determined by normalization to a reference sample of pure water of 1 $\mathrm{cm}$ thick. The scattering power of the water sample is taken equal to 6.37 electron unit per molecule unit $\mathrm{H}_{2} \mathrm{O}$ (this value calculated at $300 \mathrm{~K}$ from equation (1) is in very good 


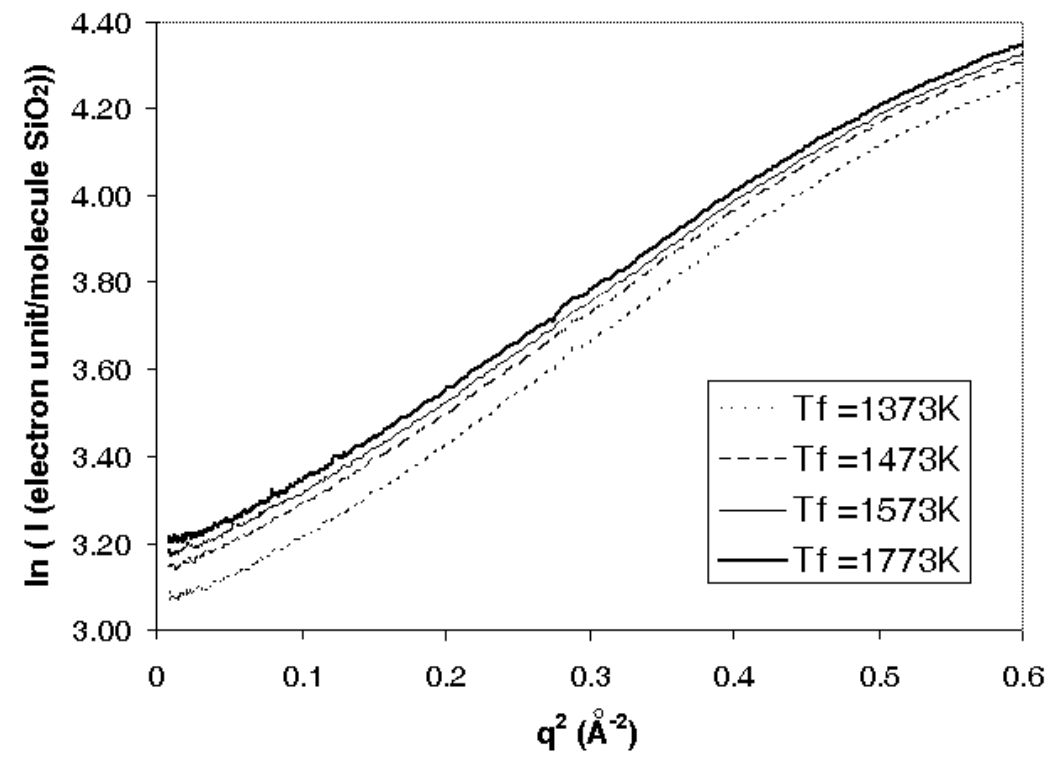

FIG. 1: Logarithm of the scattered intensity as a function of the square of the scattering vector, $q$, measured at room temperature for several silica samples with different fictive temperatures, showing a linear regime extending from $q^{2} \approx 0.04$ to $0.4 \AA^{-2}$. This linear regime is used to extrapolate the intensity towards zero scattering vector, using $I(q)=I(q=0) \exp \left(b q^{2}\right)$.

agreement with the measured value of $6.4 \pm 0.2$ electron unit per molecule $\left.\mathrm{H}_{2} \mathrm{O}\right) \underline{\underline{28}}$ The error in the final absolute intensity is estimated to be about $\pm 2 \%$.

An example of scattering curves $I(q)$ versus $q$, measured at room temperature, for four different samples, is shown on Fig. 1. The scattering curve, plotted as $\log (I(q))$ versus $q^{2}$ exhibits a linear regime extending from $q^{2} \approx 0.04$ to $0.4 \AA^{-2}$, which means that the intensity is well described by $I(q)=I(q=0) \exp \left(b q^{2}\right)$ (Ref. 8) and $I(q=0)$ is obtained as one of the regression parameters. The obtained values of $I(q=0)$ for a set of 5 samples with different fictive temperatures are shown in Fig. 2 as a function of temperature.

For one sample, three regimes can generally be identified. ${ }^{29}$ The first one is an almost linear regime in the glassy state $\left(T<<T_{g}\right)$, with a slight increase with increasing temperature. The values of intensity in this regime depends on the fictive temperature of the sample. The third regime corresponds also to a quasi-linear increase but with a larger slope in the supercooled liquid state $\left(T>T_{g}\right)$. In this regime, $I(q=0)$ values are independent of fictive 


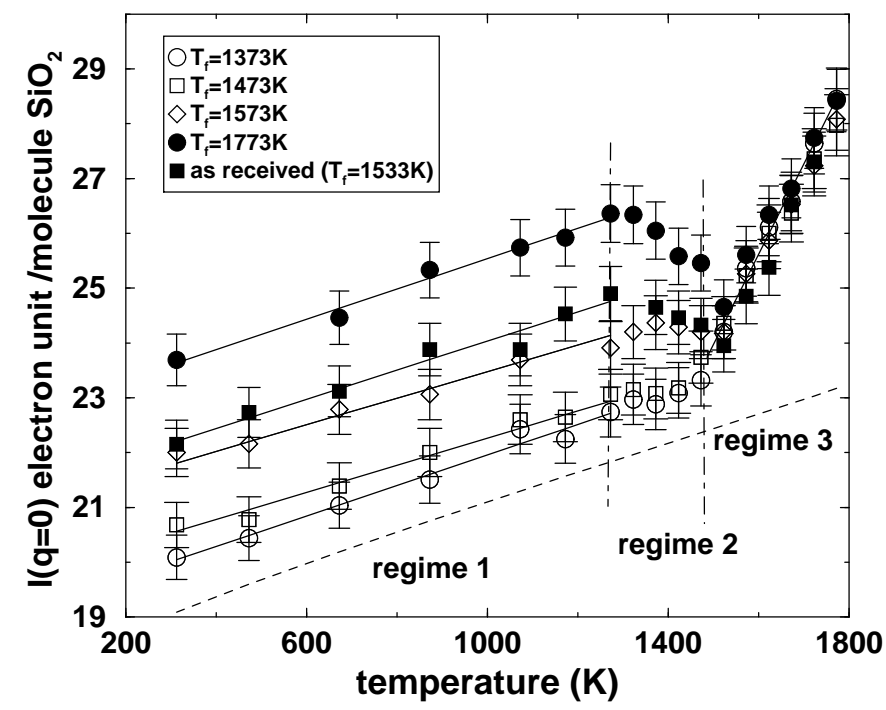

FIG. 2: Scattered intensity extrapolated to $\mathrm{q}=0$ as a function of temperature, for five samples having different fictive temperatures. The temperature dependent term due to phonons, $I^{\mathrm{ph}}$, calculated from Brillouin measurements of Ref 2, is plotted (dashed line) to show the slope of this contribution versus temperature. It is shifted of 18 e.u./molecule for clarity.

temperature within experimental errors. In the intermediate temperature range around the glass transition $I(q=0)$ follows a "structural relaxation" regime. The shape of curves in this second regime depends on $T_{f}$ as well as on the acquisition time.

\section{COMPRESSIBILITY}

In this section we present compressibility results obtained with a full point by point analysis. From our set of SAXS measurements as a function of temperature and fictive temperature we analyze the extrapolated SAXS intensity values, $I(q=0)$, in two regions. In the first one corresponding to the glassy state regime (regime 1), we used relation (10). In the second one corresponding to the supercooled liquid (regime 3 as defined in previous paragraph), relation (11) is used. Both regions corresponds to almost linear regimes for the intensity $I(q=0)$ versus temperature. The compressibility cannot be determined in the structural relaxation regime (regime 2), because the fictive temperature in that region is not known. Indeed, in this regime, it is assumed that the fictive temperature can evolve during the time of the measurement. 


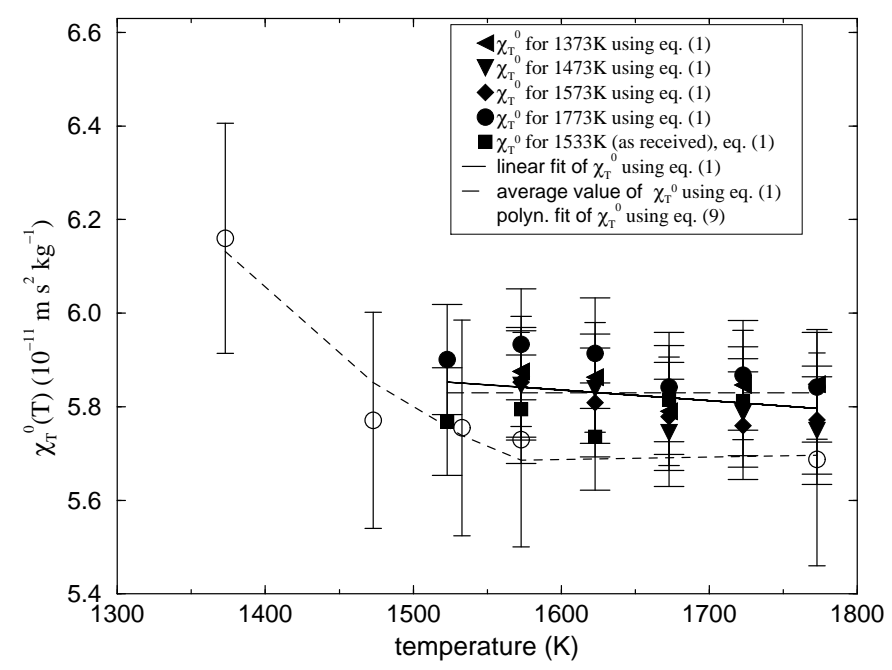

FIG. 3: Isothermal compressibility at equilibrium, determined at $T_{f}$ (filled symbols) for five silica samples having different thermal histories. $\chi_{T}^{0}$ has been determined using equation (11) in the liquid state and using equation (10), taking into account $M_{\infty}\left(T_{f}\right)$ deduced from Brillouin scattering measurements, in the glass state. The solid line in the supercooled liquid range, $1373-1773 \mathrm{~K}$, is a linear fit of the five sets of data above $1473 \mathrm{~K}$. The dot-dashed line is a quadratic fit, shown as a guide for eyes, of $\chi_{T}^{0}\left(T_{f}\right)$ in the glass state. The long dashed line is the average of the values in the supercooled liquid, obtained from the point by point analysis. For clarity, the error bars have been plotted only for one sample in the supercooled liquid.

In the liquid state, above $T_{g}$, the isothermal intensity is simply calculated for each data point by dividing the extrapolated SAXS intensity by the temperature of the measurement:

$$
\chi_{T}^{0}=\frac{I(q=0)}{T \times N_{v} k_{b}\left(\sum Z\right)^{2}},
$$

using $\sum Z=30, \rho_{0}=2200 \mathrm{~kg} \mathrm{~m}^{-3}, m=60 \times 10^{-3} \mathrm{~kg} \mathrm{~mol}^{-1}$. The error on the temperature in this range is less than $\pm 0.1 \%$, and consequently the error on the compressibility is assumed to be equal to the error on the intensity $I(q=0)$, that is about $\pm 2 \%$. The values of $\chi_{T}^{0}$ obtained from the $I(q=0)$ values for the different silica samples above $T_{g}$ are plotted as a function of temperature in fig. 3, $\chi_{T}^{0}$ appears to decrease slightly with temperature though the variations are within the errors bars. The mean value of $\chi_{T}^{0}$ calculated in this temperature range for the different silica samples is $5.82 \pm 0.12 \times 10^{-11} \mathrm{~m} \mathrm{~s}^{2} \mathrm{~kg}^{-1}$.

In order to determine the different contributions to density fluctuations in the glassy 
state, we use equation (10), where terms 1 and 2 have been combined:

$$
\begin{array}{ccc}
I(q=0) & =k_{b} N_{v}\left(\sum Z\right)^{2}\left[\chi_{T}^{0}\left(T_{f}\right)-M_{\infty}^{-1}\left(T_{f}\right)\right] T_{f} & +k_{b} N_{v}\left(\sum Z\right)^{2}\left[M_{\infty}^{-1}(T)\right] T \\
= & I^{\mathrm{res}} & +I^{\mathrm{ph}} .
\end{array}
$$

We first focus on the temperature dependent term of equation (10), $I^{\mathrm{ph}}$. This contribution (term 3), related to the longitudinal phonons, can be calculated independently of our SAXS measurements from Brillouin light scattering experiments. In order to determine the longitudinal velocity at the precise temperatures at which the SAXS measurements have been performed, we interpolate our previous high resolution Brillouin measurements, ${ }^{2}$ performed from 300 to $1773 \mathrm{~K}$ using the following polynomial fit:

$$
V_{l, \infty}(\mathrm{m} / \mathrm{s})=5788+0.6578 \times T-0.0001844 \times T^{2} \pm 0.5
$$

The sound velocity has been calculated from the Brillouin shift using a temperature dependence of refractive index. We used a linear variation of the index $n$ with temperature which does not differ from more than $0.2 \%$ from the temperature variations $\Delta n$ measured by Brückner. ${ }^{15}$ The temperature dependent index estimated by Polian et al ${ }^{14}$ is rather similar to that of Brückner up to $973 \mathrm{~K}$ but the index calculated by Polian is smaller above. The maximum difference is about $\pm 0.25 \%$ (and this would lead to less than about $\pm 0.5 \%$ difference on the determination of the intensity $\left.I^{\mathrm{ph}}\right)$. In order to calculate $M_{\infty}^{-1}$, we used a temperature dependent density determined using either measurements of specific volume by Brückner or experimental values of the linear thermal dilatation compiled by him. ${ }^{15}$ Both methods yield to density which vary from less than $0.2 \%$ in the considered temperature range and the differences on $M_{\infty}^{-1}$ and $I^{\mathrm{ph}}$ are accordingly negligible. The error on $I^{\mathrm{ph}}$ is about $0.5 \%$ ( $0.1 \%$ on the temperature, $0.4 \%$ on $\left.M_{\infty}^{-1}\right)$. Moreover, the variation of $V_{l, \infty}$ with fictive temperature is less than $0.6 \%\left(1.2 \%\right.$ on $\left.M_{\infty}^{-1}\right)$.

$I^{\mathrm{ph}}$ increases with temperature, from 1 electron unit/molecule at room temperature to 4 electron units/molecule at $1300 \mathrm{~K}$, as observed in Fig. 4. The contribution $I^{\mathrm{ph}}$ is rather small, $5-20 \%$ of $I(q=0)$. This result correlate rather well with that of Laberge which mentioned that this term contribute to about $8 \%$ of the total scattered intensity $\stackrel{10}{*}$ However, the increase of this term should be responsible for the increase of $I(q=0)$ with increasing temperature in the glassy state, as stated by Laberge. The variation of $I^{\mathrm{ph}}$ with fictive 

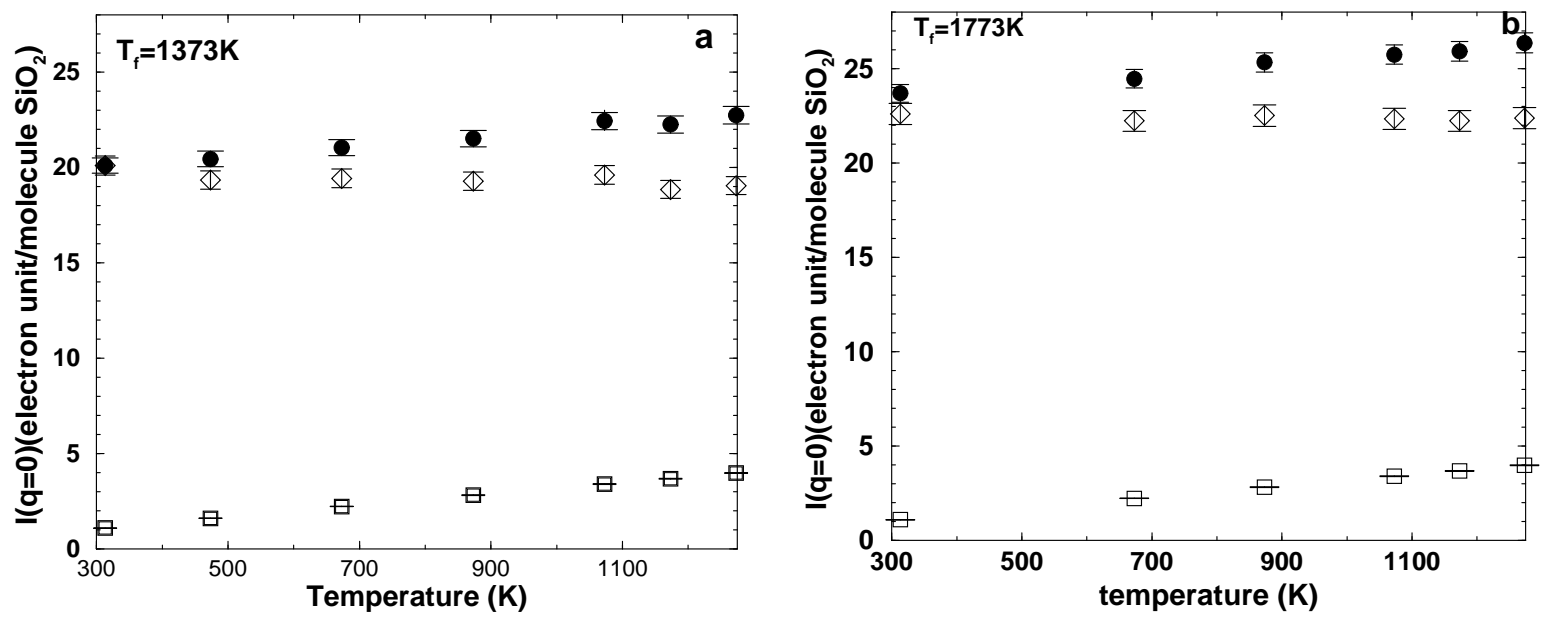

FIG. 4: (a) Scattered intensity extrapolated to $q=0(\bullet), I(q=0)$, in the supercooled liquid state for the sample of lowest fictive temperature, $T_{f}=1373 \mathrm{~K}$. The two contributions of equation (10), $I^{\mathrm{ph}}(\square)$ due to phonons (term 3) and $I^{\text {res }}(\circ)$ (related to terms 1 and 2) are also plotted. (b) Same quantities for the sample with the higher fictive temperature, $T_{f}=1773 \mathrm{~K}$.

temperature has been neglected because it amounts to less than $0.25 \%$ of the total intensity because of the small contribution of the phonons term to $I(q=0)$.

Subtracting $I^{\mathrm{ph}}$ from the total extrapolated SAXS intensity in the glassy state gives access to the residual contribution, proportional to $\left[\chi_{T}^{0}\left(T_{f}\right)-\left(M_{\infty}\left(T_{f}\right)\right)^{-1}\right] T_{f}$. For a given fictive temperature, $I^{\text {res }}$ very slightly decreases with temperature, by about 2 to $5 \%$ from 300 to $1300 \mathrm{~K}$, depending on the sample. This decrease is systematic for the 5 different fictive temperature silica samples that we analyzed, but at the limit of accuracy of the experiment. However, we note that other methods of analysis (for example using $\chi_{S}^{\infty}$ for the temperature dependent term) would yield to a stronger decrease. $I(q=0), I^{\mathrm{ph}}$ and $I^{\text {res }}$ are presented on Fig. 4 for $T_{f}=1373 \mathrm{~K}$ (Fig. 4 (a)) and for $T_{f}=1773 \mathrm{~K}$ (Fig. 4(b)).

The residual contribution, $I^{\text {res }}$, yields $\chi_{T}^{0}\left(T_{f}\right)-M_{\infty}^{-1}\left(T_{f}\right)$. The accuracy on the residual contribution is $\pm 2.5 \%$, but the error in fictive temperature $( \pm 10 \mathrm{~K})$ has to be taken into account to estimate the error on $\chi_{T}^{0}\left(T_{f}\right)-M_{\infty}^{-1}\left(T_{f}\right)$ which is then $\pm 3.5 \%$. Then $\chi_{T}^{0}\left(T_{f}\right)$ can be calculated in the glassy state using $M_{\infty}^{-1}\left(T_{f}\right)$, deduced from Brillouin measurements. $\chi_{T}^{0}\left(T_{f}\right)$, also presented in Fig. 3, varies between $6.16 \times 10^{-11} \mathrm{~m} \mathrm{~s}^{2} \mathrm{~kg}^{-1}$ for $T_{f}=1373 \mathrm{~K}$ to $5.69 \times 10^{-11} \mathrm{~m} \mathrm{~s}^{2} \mathrm{~kg}^{-1}$ for $T_{f}=1773 \mathrm{~K}$, decreasing by $8 \%$ in total with increasing fictive temperature. The error bar is $\pm 4 \%\left( \pm 0.24 \times 10^{-11} \mathrm{~m} \mathrm{~s}^{2} \mathrm{~kg}^{-1}\right)$. The values of $\chi_{T}^{0}\left(T_{f}\right)$ 
obtained in the glass state are in rather good agreement with the values obtained for the supercooled liquid. All the values are compatible with a smooth decrease of isothermal compressibility with temperature.

In order to understand the different contributions to the isothermal compressibility in the glassy state, as well as for comparison with data of the literature, it is interesting to calculate the infinite frequency and relaxational compressibilities. The infinite frequency compressibility can be determined from our previous Brillouin scattering measurements ${ }^{2}$ using equation (8). For this, we need a precise determination of the high frequency transversal velocity. We used the same temperature dependence for the refractive index as for the longitudinal velocity and found that our data in the $300-1773 \mathrm{~K}$ range can be well described by:

$$
V_{t, \infty}(\mathrm{m} / \mathrm{s})=3689+0.2946 \times T-0.0001014 \times T^{2} \pm 30
$$

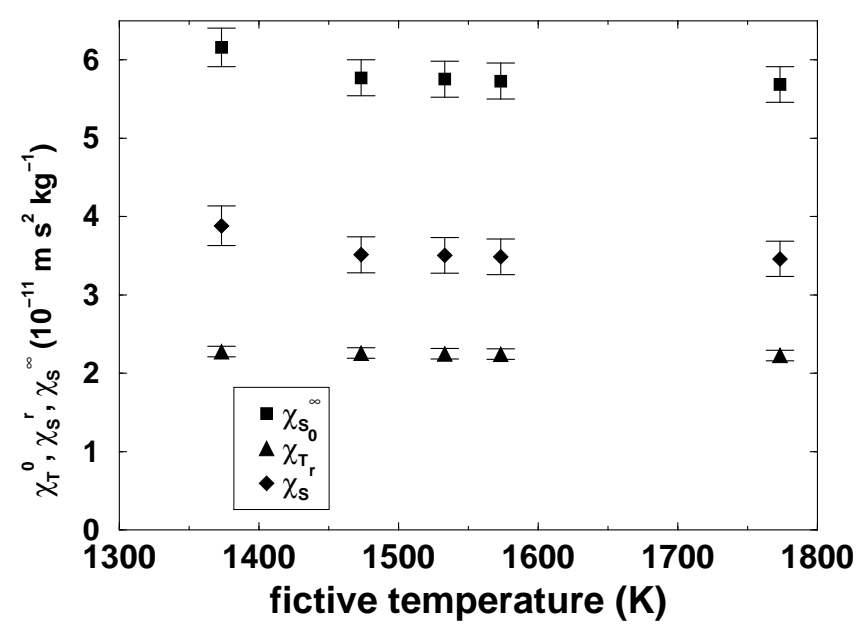

FIG. 5: Isothermal compressibility in the glass state $\chi_{T}^{0}\left(T_{f}\right)$ as a function of fictive temperature, together with the infinite frequency compressibility $\chi_{T}^{\infty}\left(T_{f}\right)$ and the relaxational compressibility $\chi_{T}^{r}\left(T_{f}\right)$.

The infinite frequency compressibility, is calculated from the combination of the experimental determinations of longitudinal and transverse velocity using equation (8). The error bar on the infinite frequency compressibility is estimated to be about $3 \%(0.2 \%$ on $\rho, 1 \%$ on the square of longitudinal sound velocity, $1.8 \%$ on the square of transverse velocity) $i$. $e$. $0.07 \times 10^{-11} \mathrm{~m} \mathrm{~s}^{2} \mathrm{~kg}^{-1}$. The error is larger on transverse velocity than on longitudinal one 
because it has to be measured using right angle geometry instead of backscattering used for longitudinal velocity. We have neglected the very low variations of the transverse and the longitudinal velocities with fictive temperature. The infinite frequency compressibility can also be fitted by a quadratic expression in $T$, valid in the $300-1773 \mathrm{~K}$ range:

$$
\chi_{S}^{\infty}\left(\mathrm{m} \mathrm{s}^{2} \mathrm{~kg}^{-1}\right)=2.917 \times 10^{-11}-7.309 \times 10^{-15} \times T+1.925 \times 10^{-18} \times T^{2} \pm 0.07 \times 10^{-11} .
$$

At the high frequency of Brillouin scattering measurements, the samples are in equilibrium and we calculate $\chi_{S}^{\infty}\left(T_{f}\right)$ using the values of $\chi_{S}^{\infty}(T)$ given by equation (19) at $T=T_{f}$. Those values are plotted in Fig. $\left[\right.$ as a function of $T_{f} . \quad \chi_{S}^{\infty}$ slightly decreases with fictive in this range we analyzed here: from $2.28 \pm 0.07 \times 10^{-11} \mathrm{~m} \mathrm{~s}^{2} \mathrm{~kg}^{-1}$ at $1373 \mathrm{~K}$ to $2.23 \pm 0.07 \times 10^{-11} \mathrm{~m} \mathrm{~s}^{2} \mathrm{~kg}^{-1}$ at $1773 \mathrm{~K}$, corresponding to a $2 \%$ decrease. $\chi_{S}^{r}\left(T_{f}\right)$ is then obtained by subtracting $\chi_{S}^{\infty}\left(T_{f}\right)$ to $\chi_{T}^{0}\left(T_{f}\right) \simeq \chi_{S}^{0}\left(T_{f}\right)$ for the five samples with different fictive temperatures. The error on $\chi_{S}^{r}\left(T_{f}\right)$ is estimated to be within $6.5 \%$. The results are shown in Fig. [5. The relaxational contribution varies from $3.88 \pm 0.25 \times 10^{-11} \mathrm{~m} \mathrm{~s}^{2} \mathrm{~kg}^{-1}$ for $T_{f}=1373 \mathrm{~K}$ to $3.46 \pm 0.23 \times 10^{-11} \mathrm{~m} \mathrm{~s}^{2} \mathrm{~kg}^{-1}$ for $T_{f}=1500^{\circ} \mathrm{C}$. It is then slightly decreasing with fictive temperature, by about $10 \%$ in the 1373-1773 range. In Fig. 5 , one can see that the infinite frequency compressibility contributes to about $40 \%$ of the static isothermal compressibility whereas the dominant contribution is the relaxational one, which amount to around $60 \%$. Our value of the relaxational compressibility is somewhat smaller than the other determinations of table I. However, the higher of those determinations from the literature $\underline{\underline{13}}$ identified as $\chi_{T}^{r}\left(T_{f}\right)$ by the authors, is rather equal to $\chi_{T}^{0}\left(T_{f}\right)-M_{\infty}^{-1}\left(T_{f}\right)$ if we follow equation (101). Thus we compared their value directly to our value of $\chi_{T}^{0}\left(T_{f}\right)-M_{\infty}^{-1}\left(T_{f}\right)$ which is equal to $5.03 \times 10^{-11} \mathrm{~m} \mathrm{~s}^{2} \mathrm{~kg}^{-1}$ for the sample of $T_{f}=1373 \mathrm{~K}$, decreasing down to to $4.57 \times 10^{-11} \mathrm{~m} \mathrm{~s}^{2} \mathrm{~kg}^{-1}$ for the others samples. The discrepancy is then smaller.

\section{DISCUSSION}

The first result of this paper is a new determination of the isothermal compressibility, obtained using small angle X-ray scattering. Most of the measurements of the isothermal compressibility quoted in the literature (see table 【) have been established using light scattering. It can be seen that the results for isothermal compressibility are rather dispersed. Our results are in agreement with previous one within error bars, though slightly lower than 
most of them. Part of the differences can occur from the experimental uncertainty on intensity measurements using light scattering technique. Indeed, any defect in the sample, such as unperfect surface polishing, bubbles and so on, can contribute in an extrinsic way to the elastic scattering. Measurements of intensity using light scattering are even more difficult at high temperature (due to thermal radiation which increases the noise). Another difficulty with light scattering measurements is that it requires large samples, in order to minimize the parasitic contribution from the surface. And on large samples, the homogeneity of the fictive temperature is difficult to achieve. Bucaro et Dardy ${ }^{11}$ estimated an error of about $\pm 3 \%$ on their measurements of $R_{L P}$ ratio. Krol et al ${ }^{12}$ mentioned that several determinations of the Landau Placzek ratio differ from less than 2\%. From our own measurements, we estimate that the Landau-Placzek ratio is usually measured with an accuracy of at least \pm 1 i.e. $\pm 4 \%$ for silica at room temperature. To illustrate this we calculated, using equation (12), the isothermal compressibility starting from Landau-Placzek ratio measured with a matching index liquid. ${ }^{30}$ We obtain $\chi_{T}^{0}=9.8 \pm 3 \times 10^{-11} \mathrm{~m} \mathrm{~s}^{2} \mathrm{~kg}^{-1}$. The error bar contains dispersion of the measurements of different fictive temperatures and differences between two sets of measurements on the same series of samples. This value illustrates that light scattering data are in agreement with our SAXS measurements for determination of the isothermal compressibility, but induce much larger errors.

The second result is the determination of the relaxational compressibility using a combination of the present SAXS data and previous Brillouin scattering measurements. This value is compared to data of the literature in column 2 of table \. Those data are calculated from isothermal compressibility in column 1 and infinite frequency compressibility shown in column 3. All the values of $\chi_{S}^{\infty}$ are in relative agreement except that of Watanabe, ${ }^{13}$ the only one not determined from Brillouin light scattering. Brillouin scattering is very accurate for the determination of the infinite frequency compressibility because it relies on the position of Brillouin lines. The differences in the values of infinite frequency compressibilities obtained from Brillouin scattering (less than $0.5 \times 10^{-11} \mathrm{~m} \mathrm{~s}^{2} \mathrm{~kg}^{-1}$ ) can be assigned to either differences in samples (for example different $\mathrm{OH}$ contents ${ }^{31}$ ) or to differences in the density or refractive index used for the analysis (the index values used in the different papers differ from up to $0.2 \%$ ). Except from our value, slightly lower, as the isothermal compressibility, all the values of relaxational compressibilities are rather close, but that of Watanabe relies on a very different value of infinite frequency compressibility. This point will be discussed 
below.

The third result is a new information brought out by our measurements: the combination of measurements below and above the glass transition to determine the isothermal compressibility as a function of fictive temperature and temperature, respectively. Two different methods of analysis were used : we performed in both cases a full point by point analysis using equation (11) in the supercooled liquid state on the one hand and using equation (10) in the glassy state on another hand. Previous studies consider either equation (11) above the glass transition, or equation (10), often applied at room temperature, assuming that the infinite frequency inverse modulus and the isothermal compressibility $11,13,17$ do not depend on temperature. A linear behavior is consequently obtained for the intensity as a function of $T$ or $T_{f}$ and the different contributions to compressibility are then obtained by a global analysis using linear regressions. $\stackrel{13,17}{17}$ The error bars are smaller than using a point by point analysis. However, by using this point by point analysis we avoid any assumption on the temperature and fictive temperature dependence of the different compressibility contributions.

For the fictive temperature dependence in the glass state, to our knowledge, the influence of thermal history on the isothermal and relaxational compressibilities have not been reported in the literature. Indeed, Saito et al have shown an influence of thermal history on the density fluctuations but their analysis rely on the assumption that the compressibility do not depend on the fictive temperature. Nevertheless, we found a rather noticeable influence of heat treatment on the isothermal compressibility with a variation of $8 \%$ for $T_{f}$ varying between $1373 \mathrm{~K}$ and $1773 \mathrm{~K}$. The changes are specially noticeable for the low $T_{f}$ (when $T_{f}$ is lowered from 1473 to $1373 \mathrm{~K}$ ). The error bar on $\chi_{T}^{0}$ being around $4 \%$, we considered this result as significant though at the limit of accuracy. This decrease with $T_{f}$ is a thoroughly new result. Such dependence was suggested by Le Parc et al ${ }^{24}$ when they discussed the fictive temperature dependence of the Rayleigh intensity in silica at room temperature. They suggest that such a dependence could explain a non linear dependence of the Rayleigh intensity, $I_{\text {Rayleigh }}$, with $T_{f}$, because $I_{\text {Rayleigh }} \propto \chi_{T}^{0}\left(T_{f}\right) \times T_{f}$. The variation of isothermal compressibility which occurs mostly at low fictive temperature is in qualitative agreement with the variations of $R_{L P}$ with $T_{f}{ }^{32}$

About the temperature dependence, the fact that $\chi_{T}^{0}\left(T_{f}\right)$ decreases with increasing fictive temperature, is a very strong support for a decrease of the isothermal compressibility 
at least in the 1373-1773 $\mathrm{K}$ range. We found almost the same temperature dependence of $\chi_{T}^{0}(T)$ in the supercooled liquid state, than that of $\chi_{T}^{0}\left(T_{f}\right)$ in the same temperature range (above $1530 \mathrm{~K})$, suggesting that $\chi_{T}^{0}(T)$ will follow the variation of $\chi_{T}^{0}\left(T_{f}\right)$ also below $1530 \mathrm{~K}$. Several values of the literature for compressibilities correspond to determinations in the glass, $\chi_{T}^{0}\left(T_{f}\right)$, obtained using equation (10), around $1400 \mathrm{~K}$ for most samples. $10,12.13$ Most of them are above the value of $5.82 \pm 0.12 \times 10^{-11} \mathrm{~m} \mathrm{~s}^{2} \mathrm{~kg}^{-1}$ found in the supercooled liquid. This also suggests a decrease with increasing temperature of the isothermal compressibility. Thus we want to emphasize that the analysis performed in the glassy state and that performed in the liquid state are in very good agreement and they both suggest a decrease of the compressibility with temperature. The small differences observed for example between $\chi_{T}^{0}\left(T_{f}\right)$ for $T_{f}=1373 \mathrm{~K}$ calculated using equation (10) and the isothermal compressibility of the same sample in the liquid are compatible with a smooth decreases of $\chi_{T}^{0}$ with temperature in the range of the glass transition. Such smooth decreases of $\chi_{T}^{0}$ with temperature would be in contrast with the data of Saito et al ${ }^{16}$ which found an isothermal compressibility constant within glassy and supercooled state but with a discontinuity and a strong increase at the glass transition. We did not observe any strong discontinuity of the isothermal compressibility at the glass transition.

A variation of isothermal compressibility with both temperature and fictive temperature induces that the global analysis by linear fitting is a rather crude approximation. In order to evaluate the impact of the approximation made using this linear fitting, we will now use such an analysis on our SAXS data. As we have measured both temperature and fictive temperature dependence of the SAXS intensity, we can plot the intensity $I(q=0)$ either as a function of $T$ or $T_{f}$ and then use linear regressions or as a function of $T$ or $T_{f}$.

Using linear fits of $I(q=0)$ a function of $T$, we can first deduce a value of the isothermal compressibility $\chi_{T}^{0}$, supposed to be independent of temperature using equation (11) for temperatures above the glass transition. We obtain $\chi_{T}^{0}=5.52 \pm 0.22 \times 10^{-11} \mathrm{~m} \mathrm{~s}^{2} \mathrm{~kg}^{-1}$. This value is very slightly lower, but close to the values obtained by the point by point analysis. Below the glass transition, we can deduce $M_{\infty}^{-1}$ from the slope and $\chi_{T}^{0}-M_{\infty}^{-1}$ from the intercept of the fitting straight line. We found that a linear law can fit the data and the slope yields a high frequency inverse longitudinal modulus $M_{\infty}^{-1}=9.16 \pm 1.5 \times 10^{-12} \mathrm{~m} \mathrm{~kg}^{-1} \mathrm{~s}^{2}$. This value is the average of the slopes obtained for each of the five samples (with different $T_{f}$ ), the error includes differences between the determinations for different samples 
as well as the error on the fitting procedure. This value is a bit lower than that determined from our Brillouin scattering, from $1.27 \times 10^{-11} \mathrm{~m} \mathrm{~kg}^{-1} \mathrm{~s}^{2}$ at room temperature to $1.12 \times 10^{-11} \mathrm{~m} \mathrm{~kg}^{-1} \mathrm{~s}^{2}$ at $1700 \mathrm{~K}$, although it is in agreement with it, taking into account the error bar. The variation with temperature of $M_{\infty}^{-1}$ determined using Brillouin measurements, by 13\%, is below the error bar on the intercepts determined from SAXS. One can also verify that the value obtained using the slope of SAXS data as a function of temperature is much closer to $M_{\infty}^{-1}$ (Ref. 10, 11, 12,, 33 ) than to $\chi_{S}^{\infty}$. These results consequently confirm the validity of equation (10), where the temperature dependent term below $T_{g}$ is governed by $M_{\infty}^{-1}$ rather than $\chi_{S}^{\infty}$. The quantity $\chi_{T}^{0}-M_{\infty}^{-1}$, deduced from the intercept $T_{f}\left[\chi_{T}^{0}\left(T_{f}\right)-M_{\infty}^{-1}\left(T_{f}\right)\right]$ of the same straight line used to determine $M_{\infty}^{-1}$, slightly decreases with increasing $T_{f}$, from $5.15 \times 10^{-11} \mathrm{~m} \mathrm{~kg}^{-1} \mathrm{~s}^{2}$ for $T_{f}=1373 \mathrm{~K}$ to $4.7 \times 10^{-11} \mathrm{~m} \mathrm{~kg}^{-1} \mathrm{~s}^{2}$ for $T_{f}=1773 \mathrm{~K}$. Those values are lower than the intercept obtained by Watanabe (table 【) using the same measuring technique and the same analysis. Indeed, as stated above, the intercept of Watanabe, corresponds, using our analysis with equation (10), to $\chi_{T}^{0}\left(T_{f}\right)-M_{\infty}^{-1}$ and not to $\chi_{T}^{r}\left(T_{f}\right)$, as he assumed. One possible reason for his higher value could rely on the measurements themselves: the absolute SAXS intensity measured by Watanabe et al. are also slightly higher than the values usually reported in silica.4,28.34.35.36 The values in the literature (except that of Ref. 13) are all around 18 and 25 electron units per molecule $\left(4\right.$ and $5.5 \times 10^{23}$ electron unit per $\mathrm{cm}^{3}$ ), whereas the data of Watanabe et al are between 5.5 and $7.12 \times 10^{23}$ electron unit per $\mathrm{cm}^{3}$. This difference could arise either from differences in the sample (different purities or different thermal histories) or from different normalization procedure for SAXS data (Watanabe normalized his SAXS data using the compressibility of quartz, Renninger used a Ludox solution, we used pure water). From the values of $\chi_{T}^{0}\left(T_{f}\right)-M_{\infty}^{-1}$, one can calculated the isothermal compressibility, by adding $M_{\infty}^{-1}$ deduced from our Brillouin scattering data, or the relaxational compressibility by subtracting $\frac{4 G_{\infty}}{M_{\infty}\left(3 M_{\infty}-4 G_{\infty}\right)}$. One obtains then $\chi_{T}^{0}$ and $\chi_{S}^{r}$ values which decrease with temperature from $\chi_{T}^{0}=6.3 \times 10^{-11} \mathrm{~m} \mathrm{~kg}^{-1} \mathrm{~s}^{2}$ and $\chi_{S}^{r}=4 \times 10^{-11} \mathrm{~m} \mathrm{~kg}^{-1} \mathrm{~s}^{2}$ for $T_{f}=1373 \mathrm{~K}$ to $\chi_{T}^{0}=5.8 \times 10^{-11} \mathrm{~m} \mathrm{~kg}^{-1} \mathrm{~s}^{2}$ and $\chi_{S}^{r}=3.6 \times 10^{-11} \mathrm{~m} \mathrm{~kg}^{-1} \mathrm{~s}^{2}$ for $T_{f}=1773 \mathrm{~K}$. The results for the isothermal and relaxational compressibilities using this method are nearly identical to that of the point by point analysis. In summary, the linear analysis as a function of temperature yield results in agreement with our point by point analysis, though it is less accurate. 
Using our SAXS data measured for the different fictive temperatures, we can also analyze the data using equation (10) as a function of fictive temperature for a given temperature of measurement. Now term 3 is constant and terms 1 and 2 vary with $T_{f}$. If we performed this analysis as a function of fictive temperature for different temperatures of measurement, we found a reasonable fit by a straight line, but the obtained $M_{\infty}^{-1}$ values are much too high (3 to 7 times too high) and varies much faster than what is obtained using Brillouin scattering measurements. Indeed, this analysis assumes that $\chi_{T}^{0}\left(T_{f}\right)-M_{\infty}^{-1}$ does not vary with $T_{f}$. It is equivalent to a linear extrapolation down to $T_{f}=0$ of data measured for $T_{f}$ around 1300 to $1800 \mathrm{~K}$. Consequently it induces large errors, specially because there is not reason why the intensity as a function of $T_{f}$ should be linear. From our point by point analysis, we observe that $\chi_{T}^{0}$ vary with temperature and $T_{f}$, and so does $\chi_{T}^{0}\left(T_{f}\right)-M_{\infty}^{-1}$. There is consequently no reason to use a linear extrapolation of $I(q=0)$ with $T_{f}$.

Having shown the uncertainty of compressibility determination using intensity measurements by light scattering and the limitations of a global analysis using linear fits of $I(q=0)$ determined by SAXS versus $T_{f}$, we come back to a comparison of our work with other small-angle measurements. Table III shows several determinations from x-ray small-angle measurements and one from neutron small-angle x-ray scattering. In each line of table III the first value of $\chi_{T}^{0}\left(T_{f}\right)$ is determined from the intensity measured at room temperature using equation (10) with $M_{\infty}^{-1}(300 K)=1.27 \times 10^{-11} \mathrm{~m} \mathrm{~s}^{2} \mathrm{~kg}^{-1}$ in term 3 and $M_{\infty}^{-1}\left(T_{f}\right) \simeq 1.12 \times 10^{-11} \mathrm{~m} \mathrm{~s}^{2} \mathrm{~kg}^{-1}$ in term 1 and 2 . The fictive temperature is either given by the authors either determined using viscosity data from Hetherington 37 and a value of $10^{12} \mathrm{~Pa}$. $\mathrm{s}$ for the viscosity at the glass transition $\left(T_{g}=1473 \mathrm{~K}\right.$ for infrared Vitreosil or $1400 \mathrm{~K}$ for O. G. Vitreosil). The values in next column is the value given by the authors themselves, when appropriate. For Ref. 13 and 38 this values used $\chi_{S}^{\infty}$ in equation (10) instead of $M_{\infty}^{-1}$, which yields to an overestimate of the isothermal compressibility. Several determinations of SAXS intensity at room temperature using equation (10) are very close to our determination. ${ }^{28.35}$ The compressibility value deduced from Ref. 38 is a bit higher but has a rather large error bar. A few determinations are slightly higher than ours..$^{4.13}$ Differences in the studied silica samples (different impurity contents, different thermal history) and uncertainty on the glass transition temperature could be partly responsible for the differences. Differences up to 0.7 to $0.8 \times 10^{-11} \mathrm{~m} \mathrm{~s}^{2} \mathrm{~kg}^{-1}$ i.e. $10 \%$ can be expected when the fictive temperature vary by $400 \mathrm{~K}$. Differences about $0.15 \times 10^{-11} \mathrm{~m} \mathrm{~s}^{2} \mathrm{~kg}^{-1}$ can 


\begin{tabular}{lll}
\hline \hline authors & $\chi_{T}\left(T_{f}\right)\left(\mathrm{m} \mathrm{s}^{2} \mathrm{~kg}^{-1}\right)$ & $\chi_{T}\left(\mathrm{~m} \mathrm{~s}^{2} \mathrm{~kg}^{-1}\right)$ \\
\hline & from Eq. (10) & from authors \\
Watanabe $\left(T_{f}=1230 \mathrm{~K}\right)^{13}$ & $8.95 \times 10^{-11}$ & $10.5 \times 10^{-11}$ \\
Watanabe $\left(T_{f}=1670 \mathrm{~K}\right)^{13}$ & $8.15 \times 10^{-11}$ & $10.5 \times 10^{-11}$ \\
this work and Ref. $2\left(T_{f}=1373 \mathrm{~K}\right)$ & $6.16 \pm 0.24 \times 10^{-11}$ & \\
this work and Ref. $2\left(T_{f}=1773 \mathrm{~K}\right)$ & $5.69 \pm 0.23 \times 10^{-11}$ & \\
Weinberg $\underline{\underline{4}}$ & $7.85 \times 10^{-11}$ & $7.8 \times 10^{-11}$ \\
Levelut $\left(T_{g}=1400-1473 \mathrm{~K}\right)^{28}$ & $6.65-6.75 \times 10^{-11}$ \\
Renninger $\left(T_{g}=1473 \mathrm{~K}\right)^{35}$ & $6.15 \times 10^{-11}$ & \\
Brüning GE-124 $\left(T_{f}=1535 \mathrm{~K}\right)^{36}$ & $5.85 \times 10^{-11}$ & \\
Brüning Corning $7980\left(T_{f}=1303 \mathrm{~K}\right)^{36}$ & $6 \times 10^{-11}$ & \\
Hulme (neutrons $)^{38}$ & $7.95 \pm 0.7 \times 10^{-11}$ & $8.5 \pm 0.7 \times 10^{-11}$ \\
\hline \hline
\end{tabular}

TABLE III: Isothermal compressibility at $T_{f}$ deduced from SAXS intensity measurements $I(q=0)$, using equation (9), $M_{\infty}^{-1}(300 K)=1.27 \times 10^{-11} \mathrm{~m} \mathrm{~s}^{2} \mathrm{~kg}^{-1}$ and $M_{\infty}^{-1}\left(T_{f}\right)=1.12 \times 10^{-11} \mathrm{~m} \mathrm{~s}^{2} \mathrm{~kg}^{-1}$. When the fictive temperature is not given in the paper, it is taken equal to the glass transition temperature (given by authors or deduced from Ref. 37, see text).

be expected when the $\mathrm{OH}$ content vary of $900 \mathrm{pmm} .^{36}$

\section{CONCLUSION}

To summarize, we want to emphasize that the values of $\chi_{T}\left(T_{f}\right)$ and $\chi_{T}(T)$ determined respectively from equation (10) and equation (11) present temperature dependences which are in very good agreement (Fig. 3i).

In this paper, we reported a complete determination of isothermal compressibility using SAXS measurements in several silica samples having different thermal histories. Using two different descriptions for the supercooled and the liquid state, we were able to determine the equilibrium isothermal compressibility $\chi_{T}^{0}$ as a function of temperature and as a function of fictive temperature, respectively. We obtained very comparable values from the two analysis, with the same temperature dependence. $\chi_{T}^{0}$ is observed to decrease with fictive temperature in a non-linear manner from 6.2 to $5.7 \times 10^{-11} \mathrm{~m} \mathrm{~s}^{2} \mathrm{~kg}^{-1}$ in the range 1373 to $1773 \mathrm{~K}$. We also 
determined the fictive temperature dependence of the relaxational compressibility, which is also decreasing with fictive temperature.

In order to compare our data with previous determinations of the literature, we performed different analysis on our data in order to evidence that differences can arise from the method of analysis.

We can also conclude from our full point by point analysis of data measured both as a function of temperature and fictive temperature, that Laberge description of the SAXS intensity in the glassy state is a very good approximation which leads to results coherent with analysis in the supercooled liquid state.

To conclude, our values of isothermal compressibility around 5.7 to $6.2 \times 10^{-11} \mathrm{~m} \mathrm{~s}^{2} \mathrm{~kg}^{-1}$, are compatible with most of the SAXS (or SANS) determinations, and not too far from the other previous determinations by light scattering, which are around $7 \times 10^{-11} \mathrm{~m} \mathrm{~s}^{2} \mathrm{~kg}^{-1}$, with a rather large error.

\section{acknowledgements}

We wish to thank O. Geyamond, S. Arnaud, and B. Caillot (Laboratoire de Cristallographie, Grenoble) for technical assistance, as well as J.-F. Bérar (Laboratoire de Cristallographie, Grenoble) for assistance in using beamline BM02. We also thanks ESRF staff for operating the synchrotron radiation facilities.

* Electronic address: claire.Levelut@lcvn.univ-montp2.fr

1 A. Q. Tool and C. G. Eichlin, J. Am. Ceram. Soc. 14, 276 (1931).

2 R. Le Parc and C. Levelut (2005), to be submitted.

3 U. Buchenau and A. Wischnewski, Physical Review B 70, 092201 (2004).

4 D. L. Weinberg, Physics Letters 7, 324 (1963).

5 J. Wendorff and E. W. Fischer, Kolloid-Z. u. Z.Polymere 251, 876 (1973).

6 R.-J. Roe and J. J. Curro, Macromolecules 16, 428 (1983).

7 J. J. Curro and R.-J. Roe, Polymer 25, 1424 (1984).

8 W. Wiegand and W. Ruland, Progr. Colloid Polym. Sci. 66, 355 (1979). 
9 J. Rathje and W. Ruland, Colloid Polym. Sci. 254, 358 (1976).

10 N. L. Laberge, V. V. Vasilescu, C. J. Montrose, and P. B. Macedo, J. Amer. Ceram. Soc. 56, 506 (1973).

11 J. Bucaro and H. D. Dardy, J. Applied Physics 46, 5324 (1974).

12 D. M. Krol, K. B. Lyons, S. A. Brawer, and C. R. Kurkjian, Phys. Rev. B 33, 4196 (1986).

13 T. Watanabe, K. Saito, and A. J. Ikushima, J. Applied Physics 94, 4824 (2003).

14 A. Polian, D. Vo-Thang, and P. Richet, Europhysics Letters 57, 375 (2002).

15 R. Brückner, J. Non-Cryst. Solids 5, 123 (1970).

16 K. Saito, H. Kakiuchida, and A. J. Ikushima, J. Non-Crystallin Solids 222, 329 (1997).

17 K. Saito and A. J. Ikushima, Appl. Phys. Lett. 70, 3504 (1997).

18 H. J. McSkimin, J. Applied Phys. 24, 988 (1953).

19 M. E. Fine, H. van Duyne, and N. T. Kenney, J. appl. Phys. 25, 402 (1954).

20 S. Spinner, J. Am. Ceram. Soc. 39, 113 (1956).

21 M. R. Vukcevich, J. Non-Crystallin Solids 11, 25 (1972).

22 B. Champagnon, R. Le Parc, and P. Guenot, Phil. Mag. B 82 (2002).

23 B. Champagnon, C. Chemarin, E. Duval, and R. Le Parc, J. Non-Cryst. Solids 274, 81 (2000).

24 R. Le Parc, B. Champagnon, P. Guenot, and S. Dubois, J. Non-Cryst. Solids 293-295, 366 (2001).

25 A. Agarwal, K. M. Davis, and M. Tomazawa, J. Non-Cryst. Solids 220 (1975).

26 A. E. Geissberger and F. L. Galeener, Phys. Rev. B 28, 3266 (1983).

27 Y. Soldo, J.-L. Hazemann, D. Aberdam, M. Inui, K. Tamura, D. Raoux, E. Pernot, J.-F. Jal, and J. Dupuy-Philon, Phys. Rev. B. 57, 258 (1998).

28 A. M. Levelut and A. Guignier, Bull. Soc. Franc. Mineral. Crist. 90 (1967).

29 C. Levelut, A. Faivre, R. Le Parc, B. Champagnon, J.-L. Hazemann, L. David, C. Rochas, and J.-P. Simon, J. Non-Cristallin. Solids 307-310, 426 (2002).

30 B. Champagnon (2005), private communication.

31 F. Terki, C. Levelut, M. Boissier, and J. Pelous, Phys. Rev. B 53, 2411 (1996).

32 R. Le Parc, B. Champagnon, L. David, A. Faivre, C. Levelut, P. Guenot, J.-L. Hazemann, C. Rochas, and J.-P. Simon, Philos. Mag. B 82, 431 (2002).

33 J. Shroeder, R. Mohr, P. B. Macedo, and C. J. Montrose, J. Amer. Ceram. Soc. 56, 510 (1973).

34 T. Gerber and B. Himmel, J. Non-Cristallin. Solids 83, 324 (1986). 
35 A. L. Renninger and D. R. Uhlmann, J. Non-Cryst. Solids 16, 325 (1974).

36 R. Brüning, C. Levelut, A. Faivre, R. Le Parc, J.-P. Simon, F. Bley, and J.-L. Hazemann, Europhysics Letters 70, 211 (2005).

37 G. Hetherington, K. H. Jack, and J. C. Kennedy, Phys. Chem. Glasses 5, 130 (1964).

38 R. Hulme, Ph.D. thesis, University of Reading (U.K) (1991). 